meetings and on its advice St. Dunstan's has decided to set up a Research Unit and to seek the full-time services of a research physicist and a biologist. Through the generosity of an anonymous benefactor, a substantial sum of money has been made available for the work of the Committee over a period of five years. The Committee will not only investigate guiding devices for the blind but also methods whereby the printed word in an ordinary book can 'read aloud' to the blind; the improvement of recorded talking books ; braille machines, and other apparatus. The research work will include the better use of the little glimmer of sight which remains with many people who are technically blind, and the substitution of the sense of sight by the other senses.

\section{Localization in the Visual Cortex}

Dr. Gordon Holmes, in his Ferrier Lecture before the Royal Society on "The Organisation of the Visual Cortex in Man" (Proc. Roy. Soc., B, 132, 348 ; 1945), dealt chiefly with the question of localization within the visual area of the cerebral cortex. As he himself has shown, by study of the visual-field defects resulting from gunshot wounds of the cortex, there is apparently a very sharp point-to-point representation of the retina in the cortex. The conception of definite fixed anatomical connexions between each point of the retina and the corresponding point in the cortex has been supported by recent histological studies of the actual nerve fibre connexions, particularly by Le Gros Clark. Such a rigid fixed relationship between retina and cortex would appear, however, to stand in marked contrast to the situation in other parts of the cortex, notably the motor area, where localization is by no means sharp and undergoes considerable physiological variations. But more recent clinical studies have shown that there must be a good deal of plasticity in the functional organization of the visual cortex. For example, patients suffering from hemianopia compensate for the loss of half the visual field by developing a new fixation point ('false macula') in the centre of the surviving field of vision ; a similar adaptation occurs in a squinting eye. In such cases there must be a complete functional reorganization in the cortex, though the fibre connexions, of course, cannot change. The conclusion is that, although there is an accurate point-to-point representation of the retina in the cortex, the functional organization of the visual cortex is not thereby rigidly fixed; on the contrary, it exhibits considerable plasticity.

\section{Recent Earthquakes}

During April and the early part of May 1945, the United States Coast and Geodetic Survey, in cooperation with Science Service and the Jesuit Seismological Association, determined the provisional epicentres of five earthquakes. The first two occurred on April 15, the first at 2h. 35.2m. G.M.T. and the second at $19 \mathrm{~h} .50 \cdot 6 \mathrm{~m}$. G.M.T. The former had an epicentre at lat. $56^{\circ} \mathrm{N}$., long. $164^{\circ} \mathrm{E}$., which is east of Kamchatka, and the latter an epicentre at lat. $22 \cdot 5^{\circ} \mathrm{N}$., long. $108 \cdot 0^{\circ} \mathrm{W}$., which is off Mazatlan, Mexico. The third earthquake was on April 19 at 13h. $03.5 \mathrm{~m}$. G.M.T. from an epicentre near lat. $40^{\circ} \mathrm{S}$., long. $179^{\circ} \mathrm{E}$., which is east of New Zealand. The earthquake of April 21 at 17h. 14.5m. G.M.T., according to the calculations based on reports from twelve earthquake observatories (instrumental), had its epicentre at lat. $19 \cdot 3^{\circ} \mathrm{N}$., long. $100 \cdot 6^{\circ} \mathrm{W}$. Its depth of focus was below normal, probably $50-100 \mathrm{~km}$. below the surface of the earth. The earthquake was felt in Mexico. The fifth earthquake was on May 19 at 7h. $55 \cdot 8 \mathrm{~m}$. G.M.T. The provisional epicentre, based on instrumental reports from eight observatories, has been estimated at lat. $16 \cdot 0^{\circ} \mathrm{N}$., long. $98 \cdot 4^{\circ} \mathrm{W}$., which is west of Mexico.

Mr. E. W. Pollard, at his observatory at Binstead, Isle of Wight, registered five earthquakes during April, the first on April 15 being the greatest. May is reported as being a very quiet month, only two earthquakes being recorded, on May 9 and 19. The former was small and the latter medium strength.

\section{Localization of Faults in Low-Voltage Cables}

PRACTICAI limitations of well-known fault-locating tests are considered, and some new tests capable of high accuracy are described, in a paper read by J.H. Savage recently before the Institution of Electrical Engineers in London. The new tests include a D.c. valve-voltmeter circuit for core-to-sheath insulation faults, audio-frequency search methods for open circuits and radio-frequency tests for conductor defects. Mention is also made of fault localization based on wave-reflexion effects. 'The paper deals mainly with factory technique for rubber- and plasticinsulated cables; but some of the methods can be applied to other types and to field work. As faults on cables carrying current are accompanied by local changes of electric and magnetic fields, and circuit unbalance and wave reflexions can be produced, future developments are likely to be in the direction of electronic devices, because these are eminently suitable for detecting and recording such effects.

\section{Chinese Visitors to Britain}

Eraнt Chinese visitors of widely varying professions are visiting Britain as guests of the British Council : Dr. Wang Ging-Hsi, director of the Institute of Psychology of the Academia Sinica, a well-known Chinese physiologist ; Dr. Sah Pen-Tung, president of the National University of Amoy, Fukien, who is a physicist especially interested in radio-engineering; Dr. Yang Chen-sheng, professor of Chinese literature, and acting dean of the College of Arts of the National University of Peking ; Prof. Tung $\mathrm{Li}_{\mathrm{i}}$ Yuan, librarian of the National Library, Peking; Colonel (Mrs.) Chow Mei-You, head of the Nursing School in the Army Medical School; Miss Gao Ren-Ying, acting national general secretary of the Chinese Y.W.C.A., who is studying social conditions in Britain; Miss Priscilla Huang and Miss Chi-yi Chen, both of whom are interested in children's and women's welfare and all services of rehabilitation of war casualties.

\section{Canadian Awards for Postgraduate Training}

The National Research Council of Canada is providing opportunities for postgraduate training in science for men and women whose studies have been delayed or interrupted by war service, either military or civilian. Three classes of award are available for the academic year 1945-46 as follows : bursaries $(450$ dollars) for students who have graduated with high distinction in scientific subjects; studentships $(750$ dollars) for students who have had experience in research work in science for at least one year following graduation; fellowships $(900$ dollars $)$ for students who have given distinct evidence of capacity to conduct independent research in science. One group of applications is under consideration and 